\title{
ARGENTINA EN TIEMPOS DE CUARENTENA. ACCIDENTES DE TRÁNSITO. COBERTURA PÓLIZA DE SEGUROS. PAGOS DE PRIMA. RELACIONES DE CONSUMO
}

\author{
ARGENTINA IN QUARANTINE TIMES. TRANSIT \\ ACCIDENTS. INSURANCE POLICY COVERAGE. PREMIUM \\ PAYMENTS. CONSUMPTION RELATIONS
}

\author{
FABIÁN ANTONIO GIROLIMETTO* \\ LETICIA PELLE DELGADILLO** \\ Fecha de recepción: 30 de abril 2020 \\ Fecha de aceptación 15 mayo 2019 \\ Disponible en línea: 30 de junio 2020
}

Para citar este artículo/To cite this article

Girolimetto, Fabián Antonio \& Pelle Delgadillo, Leticia. Argentina en tiempos de cuarentena. Accidentes de tránsito. Cobertura póliza de seguros. Pagos de prima. Relaciones de consumo, 52 Rev.Ibero-Latinoam.Seguros, 81-86 (2020). https://doi.org/10.11144/Javeriana.ris52.atca

Doi: 10.11144/Javeriana.ris52.atca

\footnotetext{
* Abogado - Universidad Nacional de Córdoba - Especialista en Derecho de Seguros, Responsabilidad Civil, Derecho Laboral e Infortunios Laborales, Oralidad, Mediaciones, Defensa del Consumidor, Recuperos, Fraude - Autor de Publicaciones y Ponencias en Derecho de Seguros - Secretario Académico de la Sala de Derecho de Seguros del Colegio de Abogados de Córdoba - Miembro AIDA-Rama Argentina - Síndico en la Caja de Previsión y Seguridad Social de la Provincia de Córdoba - Secretario de la Asociación para el Estudio del Derecho de Seguros del Interior Argentino (AEDSIA). Contacto: fabiangirolimetto@hotmail.com ** Abogada - Universidad Católica de La Plata - Diplomada en Derecho de Seguro por la Universidad de Ciencias Empresariales y Sociales (UCES). Autora de Publicaciones y Ponencias en Derecho de Seguros Derecho de Consumidor- Subdirectora académica del Instituto de Derecho del Consumidor del Colegio de Abogados de La Plata-Secretaria del Instituto de Derecho de Seguros del Colegio de Abogados de La Plata- Miembro AIDA -Rama Argentina - Subdirectora Instituto "Nuevas Tecnologías, prevención y seguros" AIDA- Miembro de la Asociación para el Estudio del Derecho de Seguros del Interior Argentino (AEDSIA), Miembro del Instituto Argentino de Derecho del Consumidor. Contacto: leti_pelle@hotmail.com
} 


\title{
RESUMEN
}

El régimen de cuarentena por pandemia del coronavirus impuesto por el gobierno argentino, generó vía redes sociales y declaraciones públicas, una serie de interrogantes para el común de los argentinos, sobre la posibilidad de exclusión de cobertura por parte de la aseguradora en caso de un siniestro, así como también referido al plazo y al monto de prima a pagar por los asegurados en esta época de excepción que se está viviendo.

La crisis sanitaria que atraviesa el mundo debido a la propagación del COVID 19, plantea grandes desafíos, ello sin dudas a la ciudadanía toda. Asimismo, y en consecuencia directa con la situación mundial atravesada, nos encontramos con situaciones jurídicas entre proveedores y consumidores que se ven por demás afectadas. Debiendo analizar cada caso y circunstancias concretas, contemplando los principios de buena fe, solidaridad y esfuerzo compartido.

Palabras Clave: Seguros, exclusión de cobertura, prima, COVID-19, improvisión, relación contractual, buena fe, sujetos vulnerables, protección, esfuerzo compartido, justicia.

\begin{abstract}
The coronavirus quarantine regime imposed by the Argentine government, generated via social networks and public statements, a series of questions for the common Argentines, about the possibility of exclusion of coverage by the insurer in case of a claim, as well as referring to the term and the amount of premium to be paid by the insured in this exceptional time that is being experienced.

The health crisis that the world is going through due to the spread of COVID 19 , poses great challenges, undoubtedly, both for companies and for all citizens. Likewise, and as a direct consequence of the world situation, we are faced with legal situations between suppliers and consumers that are affected by others. Must analyze each case and specific circumstances, considering the principles of good faith, solidarity and shared effort.
\end{abstract}

Key Words: Insurance, exclusion of coverage, premium, COVID-19, improvisation, contractual relationship, good faith, vulnerable subjects, protection, shared effort, justice.

\section{SUMARIO}

1. Accidentes de tránsito. Cobertura póliza de seguros. Pagos de prima-.

2. Relaciones de consumo y COVID-19. 


\section{ACCIDENTES DE TRÁNSITO. COBERTURA PÓlIzA DE SEGUROS. PAGOS DE PRIMA}

En las siguientes líneas se procura abordar algunas dudas que se han presentado en estos días, a raíz del régimen de cuarentena por pandemia del coronavirus impuesto por el gobierno argentino hasta el 31/03/2020, disposición que prohíbe la circulación, salvo determinadas excepciones. Ante dicha normativa (DNU N²97/2020 y sus reglamentaciones), se emitieron ciertas declaraciones públicas de funcionarios a nivel nacional así como mensajes a través de las redes sociales, de manera que se presentaron una serie de interrogantes para el común de los argentinos; colectivo que comenzó a plantearse dos situaciones: a) qué sucede en caso de la producción de un accidente de tránsito durante este estado de cuarentena, Así, las consultas más frecuentes de los clientes pueden sintetizarse como sigue: ¿me cubre mi seguro de Responsabilidad Civil si durante esta contingencia y ante un caso excepcional, circulo por las calles y choco? ¿me cubre mi seguro de Responsabilidad Civil si circulo sin respetar la cuarentena?, entre otras preguntas. Por otro lado, también se ha planteado la hipótesis: b) qué sucede con el pago mensual de la prima, pues los ciudadanos consultan, por ejemplo: ¿estoy obligado a pagar mi prima mensual aun cuando no se usa el vehículo con motivo de la cuarentena? ¿Cómo abono la prima mensual si no puedo salir de mi domicilio? ¿si me demoro en el pago de la prima en función de la cuarentena, se mantiene la cobertura del seguro en caso de siniestro? En esta línea, son múltiples las preguntas que nos llegan día a día motivados por la incertidumbre que se ha generado en virtud de esta situación excepcional e intempestiva que nos toca vivir en la actualidad.

Sin pretender exhaustividad, podemos señalar, respecto al primer tema, que la cuarentena decretada por el gobierno nacional no modifica la protección que ofrece el seguro que se haya contratado para un determinado automóvil, tanto en su modalidad de seguro a terceros como en el resto de las garantías. En general, no figura dentro de los contratos de seguros contratados una exclusión expresa de cobertura por pandemia o cuarentena (tampoco se prevé como supuesto no cubierto). En consecuencia, en caso de circular, los asegurados contarían con cobertura en caso de siniestro. Ello resulta independiente de la responsabilidad penal que le cabría al ciudadano en el supuesto de que la autoridad constate que incurrió en algún supuesto de violación del DNU y sus reglamentaciones. Además, un dato no menor, es que el gobierno en su DNU permite desplazamientos mínimos e indispensables a todas las personas, para adquirir productos de limpieza, medicamentos y alimentos. No obstante lo señalado en forma genérica supra, siempre aconsejamos consultar con detalle el texto de cada una de las pólizas que se hayan contratado, a los fines de verificar - de modo especial- las exclusiones previstas contractualmente. Ello desde que las exclusiones no son generales sino particulares y se analizan frente a cada caso particular. Los contratos continúan vigentes en la cuarentena. Independientemente de lo relacionado líneas atrás, no podemos dejar de puntualizar que - generalmente- al contratar un Seguro de Responsabilidad Civil, esta posibilidad se ofrece conjuntamente con la cobertura de otros riesgos, tales como incendio de la unidad, Robo/Hurto, Destrucción Total/Parcial, etc., para lo cual no se requiere la circulación del vehículo asegurado. La Superintendencia de Seguros de la Nación Argentina realizó un comunicado oficial con el objetivo de despejar algunas 
de estas dudas generadas a partir de información falsa viralizada en las redes sociales (fake news). En este sentido, el organismo ha indicado que cualquier tipo de aviso al respecto será publicado en los canales habituales y oficiales de la misma.

Respecto a la segunda cuestión planteada relacionada con el pago de la prima del contrato, debemos decir que configura una obligación del asegurado la de abonar la misma en tiempo y forma, a los fines de contar con cobertura en caso de un siniestro. En la actualidad, en la mayoría de los casos, las primas son abonadas por intermedio de débito automático a través de las tarjetas de crédito, o bien, por algún otro medio digital de pago. Tal modalidad implica que no se requiere la presencia personal ante la compañía aseguradora para efectuar el pago correspondiente. Por otro lado, en los últimos días y mediante comunicados vía redes sociales, algunas compañías aseguradoras han informado a sus clientes que se han flexibilizado los cumplimientos del pago de las primas mensuales y que los eventuales retrasos en este sentido no afectarán la cobertura ni implicarán la suspensión de la misma. Todo ello con el fin de apoyar a los clientes asegurados frente a las dificultades económicas que pudieran atravesar debido al desarrollo de los acontecimientos que son públicamente conocidos, ocasionado a raíz de la cuarentena decretada por el gobierno nacional por la pandemia del coronavirus. No obstante, pese a estos avisos a los que se hacen referencia, aconsejamos a cada uno de los ciudadanos que efectúe una consulta directa con la compañía aseguradora con la que se vinculan para confirmar las medidas adoptadas por la misma, a los fines del correcto resguardo de sus derechos.

\section{RELACIONES DE CONSUMO Y COVID-19}

La crisis sanitaria que atraviesa el mundo debido a la propagación del COVID-19, plantea grandes desafíos a la ciudadanía toda.

Desde hace décadas el derecho se ocupa de los más débiles los más vulnerables, especialmente se ocupa en la actualidad de los derechos de los Consumidores y Usuarios, mediante la protección de un conjunto de herramientas jurídicas que permitan equilibrar esa relación en principio desventajosa frente a sus proveedores.

Lo complejo de esta situación sanitaria que tristemente estamos atravesando en este momento histórico, nos lleva a afirmar que no solo los usuarios y consumidores - $\mathrm{O}$ normalmente el sujeto vulnerable de un vínculo jurídico- son quienes no pueden hacer frente a las relaciones jurídicas previamente asumidas.

Muchas empresas (entre ellas las compañías aseguradoras) han visto sustancialmente modificadas sus prestaciones, limitada o reducida su actividad por el propio contexto de emergencia sanitaria y las medidas de confinamiento de la población decretadas por el Gobierno Nacional.

Otras se han visto incluso obligadas legalmente a suspender su actividad, sin posibilidad de mantener la apertura al público de sus establecimientos o locales de confor- 
midad con lo dispuesto por los Decretos de Necesidad y Urgencia. Viéndose alterada de esta forma las contraprestaciones y los vínculos jurídicos previos a la mencionada situación sanitaria.

Asimismo, y en consecuencia directa con la situación mundial atravesada, nos encontramos con situaciones jurídicas entre proveedores y consumidores que se ven por demás afectadas.

En nuestro país, la normativa consumeril, logra una fuerte protección a quien entiende es el sujeto más débil de la relación, sin embargo, las situaciones actuales totalmente extraordinarias conllevan un análisis más profundo.

Pues bien es necesario contemplar todas las partes de esos vínculos jurídicos, debiendo ser vistas por el Estado y por el intérprete jurídico. Cobrando valor teorías que manteníamos sin demasiada aplicación, como lo es la del esfuerzo compartido.

La sanción de nuestro actual Código Civil y comercial, provee distintas soluciones según sea el caso concreto. Y por ello contempla, la frustración del fin del contrato, la modificación del contrato y la teoría de la imprevisión entre otras, en su artículo 1091.

El citado artículo expresa lo siguiente, cuando ocurra una "alteración extraordinaria de las circunstancias existentes al tiempo de su celebración...”.

Coincidimos en que la situación actual que vivimos en relación al COVID-19 resulta por lo demás excepcional e imprevista. Pudiendo en base a esta teoría, resolver y readecuar el contrato judicial o extrajudicialmente, pero en estas circunstancias, ¿cómo deberá evaluar el juzgador la responsabilidad por esas prestaciones que no llegaron a satisfacerse?

Nuestro país posee antecedentes de situaciones extraordinarias en nuestra historia económica -aunque jamás uno similar al actual-, habiendo incidido los mismos en aquellos contratos que se encontraban en curso de ejecución, por ejemplo, el llamado "Rodrigazo", la salida de la llamada "tabla de Martínez de Hoz", la hiperinflación de 1989, la salida de la convertibilidad y la sanción de la Ley $N^{\circ} 25.561$, entre otros.

La diferencia en la actualidad radica en que nuestro actual ordenamiento jurídico contempla soluciones más armoniosas para ambas partes, protegiendo especialmente al consumidor.

Para ello debemos desarrollar que alcance tiene la Teoría de la imprevisión en nuestro país, y entendemos que la misma se aplicará cuando por un evento extraordinario, sobreviniente, imprevisible torne dificultoso o excesivamente oneroso el cumplimiento de aquellas prestaciones pactadas. Por lo cual basadas en nuestro principio de buena fe, contemplado en el artículo 9 del CCYC, ambas partes deberán adecuar las condiciones primarias establecidas.

Teoría que fue concebida como una herramienta para restablecer el equilibrio contractual, no extintivo de los contratos. 
Así refiere el Dr. Profesor Walter KRIEGER ${ }^{1}$, al manifestar que en nuestro ordenamiento, el marco protectorio del consumidor, de raigambre constitucional, tienen como principios fundamentales, el Protectorio y el de Sustentabilidad.

Entendiendo que se protege a aquella parte más débil, desde el punto de vista del acceso y alcance de la información que ambas partes manejan. Es necesario considerar como herramienta fundamental a la hora de resolver estos litigios, que el dialogo de fuentes resulta ser una herramienta fundamental.

Es decir que no solo nos vamos a valer de la Ley de Defensa del Consumidor ${ }^{2}$ si no que también vamos a remitirnos a otras normas por ejemplo si analizamos la implementación del Derecho del Consumidor, en aplicación de las normativas vigentes, emergentes de la Ley especial $N^{\circ} 24.240$, en diálogo con aquellas incorporadas en el Código Civil y Comercial de la Nación, debe efectuarse siempre con la Constitución Nacional como norte (Arts. 42, 43 , e instrumentos internacionales incorporados por el Art. 75 inc. 22), y con un notorio direccionamiento en favor del consumidor, en función del principio protectorio, y aplicando las leyes complementarias que correspondan al caso concreto, como Ley de la Competencia, CCYC, etc. construyendo así soluciones particulares que permitan resolver los conflictos planteados. Entendiendo que por la particularidad y excepcionalidad e los tiempos vividos las respuestas no podrán ser globales.

Debiendo analizar cada caso y circunstancias concretas, contemplando los principios de buena fe, solidaridad y esfuerzo compartido.

Por lo cual de lo expuesto podemos concluir que, nos encontramos en una nueva realidad, impensada, sin antecedentes, y de la cual surgieron nuevos sujetos débiles a los cuales el juzgador deberá contemplar previo a sus decisiones finales, evitando abusos, propiciando la buena fe, el esfuerzo compartido, en definitiva, darles justicia, darle a cada uno lo que le corresponde.

\footnotetext{
1 http://www.fasj.org.ar/wp/2020/04/01/1 ra-conferencia-la-imprevision-en-e-derecho-de-consumidorpasajes-aereos-y-hosteleria-walter-krieger-argentina/.

2 Defensa del Consumidor, dialogo de fuentes y principio de protección Autor: STIGLITz Gabriel, Revista de Derecho del consumidor $N^{\circ} 1$ noviembre de 2016, cita IJ-CCXVIII-919.
} 